\section{An Improved Method of Automated Noise Measurement System in CT Images}

\author{
Choirul Anam*๑, Idam Arif², Freddy Haryanto², Fauzia P \\ Lestari³, Rena Widita², Wahyu S Budi', Heri Sutanto', Kus- \\ woro Adi', Toshioh Fujibuchi' ${ }^{4}$, Geoff Dougherty ${ }^{5}$
}

\begin{abstract}
Background: It is necessary to have an automated noise measurement system working accurately to optimize dose in computerized tomography $(\mathrm{CT})$ examinations.
\end{abstract}

Objective: This study aims to develop an algorithm to automate noise measurement that can be implemented in CT images of all body regions.

Materials and Methods: In this retrospective study, our automated noise measurement method consists of three steps as follows: the first is segmenting the image of the patient. The second is developing a standard deviation (SD) map by calculating the SD value for each pixel with a sliding window operation. The third step is estimating the noise as the smallest SD from the SD map. The proposed method was applied to the images of a homogenous phantom and a full body adult anthropomorphic phantom, and retrospectively applied to 27 abdominal images of patients.

Results: For a homogeneous phantom, the noises calculated using our proposed and previous algorithms have a linear correlation with $\mathrm{R}^{2}=0.997$. It is found that the noise magnitude closely follows the magnitude of the water equivalent diameter $\left(\mathrm{D}_{\mathrm{w}}\right)$ in all body regions. The proposed algorithm is able to distinguish the noise magnitude due to variations in tube currents and different noise suppression techniques such as strong, standard, mild, and weak ones in a reconstructed image using the AIDR 3D algorithm.

Conclusion: An automated noise calculation has been proposed and successfully implemented in all body regions. It is not only accurate and easy to implement but also not influenced by the subjectivity of user.

Citation: Anam Ch, Arif I, Haryanto F, Lestari FP, Widita R, Budi WS, Sutanto H, Adi K, Fujibuchi T, Dougherty G. An Improved Method of Automated Noise Measurement System in CT Images. J Biomed Phys Eng. 2021;11(2):163-174. doi: 10.31661/jbpe.v0i0.1198.

\section{Keywords}

Ionizing Radiation; X-Rays; Computed Tomography; Image Quality; Automated Noise Calculation; Algorithms; Image Processing, Computer-Assisted

\section{Introduction}

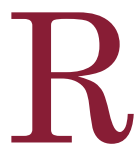

ecent studies have revealed that CT scanning poses a potential cancer risk in patients [1-4]. However, the risk has been considered much smaller than the benefits of using a CT scan to diagnose accurately patient abnormalities for effective therapies [5]. The risk must be minimized based on as low as reasonable achievable (ALARA) [6]. In the principle of ALARA, the radiation optimization is more important in comparison with radiation dose reduction. Dose reduction is relatively easy to be achieved by reduction of tube current; however, this approach decreases image quality. In radiation optimization, image quality is more important than patient dose [7]; thus, before obtaining
${ }^{1} \mathrm{PhD}$, Department of Physics, Faculty of Sciences and Mathematics, Diponegoro University, Jl. Prof. Soedarto SH, Tembalang, Semarang 50275, Central Java, Indonesia

${ }^{2} \mathrm{PhD}$, Department of Physics, Faculty of Mathematics and Natural Sciences, Bandung Institute of Technology, Ganesha 10, Bandung 40132,

West Java, Indonesia

${ }^{3} \mathrm{MSc}$, Department of

Physics, Faculty of Mathematics and Natural Sci-

ences, Bandung Institute

of Technology, Ganesha

10, Bandung 40132,

West Java, Indonesia

${ }^{4} \mathrm{PhD}$, Department of

Health Sciences, Faculty of Medical Sciences,

Kyushu University, 3-1-1

Maidashi, Higashi-ku,

Fukuoka 812-8582,

Japan

${ }^{5} \mathrm{PhD}$, Department of Applied Physics and Medi-

cal Imaging, California

State University Channel

Islands, Camarillo, CA

93012, USA

*Corresponding author: Choirul Anam

Department of Physics,

Faculty of Sciences and

Mathematics, Dipo-

negoro University, J.

Prof. Soedarto SH,

Tembalang, Semarang

50275, Central Java,

Indonesia

E-mail: anamfisika@

gmail.com

Received: 10 June 2019 Accepted: 4 August 2019 
the image using the smallest possible dose, an image of sufficient quality to diagnose the patient must be achieved.

There are a number of parameters to characterize image quality, including noise magnitude, low contrast detectability, and spatial resolution $[8,9]$. Parameters of image quality have been usually evaluated using a standard phantom of standardized size $[10,11]$. The evaluation of image quality, using the standard phantom, is very good for assessing dependency on controllable variables, such as tube currents, tube voltage, pitch, reconstruction filter, and beam width [9]. However, image quality is also influenced by uncontrollable variables, such as body size and body region [7, 12-14]. The controllable and uncontrollable variables must be considered simultaneously to optimize purposes [7].

Noise magnitude is probably the most important parameter for characterizing image quality. Measurements of noise magnitude in a clinical image, are usually performed manually based on calculating the standard deviation (SD) within a region of interest (ROI) in the most homogeneous region $[15,16]$. For a more comprehensive approach, noise is characterized using the noise power spectrum (NPS) [17-19]. However, manual measurements of noise are not practical in clinical settings, especially in busy medical centers. Moreover, the determination of a ROI manually is very subjective and influenced by the experience of the relevant medical personnel. A small shift in the position of the ROI leads into different estimates of the noise magnitude. Consequently, the development of algorithms is essential to have robustly automated noise calculations.

Recently, Christianson et al. have developed an algorithm to measure automatically the noise in clinical CT images without determining a ROI [20]. The algorithm consists of four steps as follows: 1) soft tissue segmentation based on density slicing of the CT images using Hounsfield unit (HU) values from 0 to +100 2) SD calculation for every pixel to cre- ate a SD map using a sliding window operation 3) a SD histogram developed from the SD map of the CT image and 4) the determination of the noise magnitude as the most frequent $\mathrm{SD}$ in the SD histogram. They reported that the approach successfully distinguished noise due to kernel reconstruction variations, dose variations, and slice thickness variations for three CT scanner models [20]. The result of the automated noise calculation is called the global noise level because the automated noise was identified as the most frequent SD level in soft tissues. There are two limitations to this method. The first is that the method of automated noise calculation may only be applied to the abdomen region because noise calculation is carried out by calculating the SD map on the region where the majority composition is soft tissue [20]. The second is that the global noise magnitude is an overestimate compared to the predicted noise magnitude. They reported that the average differences between automated and manual noise measurements, using the subtraction method, were $3.4 \%$ and $4.7 \%$ for phantom and clinical CT images, respectively [20]. However, many later studies reported that the subtraction of two image sets resulted in a residual noise image with twice the noise magnitude of an individual image [21-23].

More recently, Chun et al. have proposed an algorithm of automated calculation of CT noise in patient images using a structure coherence feature [24]. The algorithm used the ROIs located carefully in the coherence region; therefore, it may overcome the overestimation of global noise from the previous method [20], although they did not report any comparison with that study. The algorithm consists of four steps, including subcutaneous fat tissue segmentation, the measurement of structure coherence feature, determination of homogeneous ROIs and estimation of the noise magnitude. Although this method is not limited just to the abdomen, it cannot be applied to the head since the method determines 
a homogeneous area limited to fat that has a unique HU range, i.e. from -150 to -30 [24].

It is very important to develop an automatic calculation of noise magnitude applied generally to all parts of the body. It should not be limited to soft tissue or fat or certain other tissues. This study aims to develop an algorithm for automated noise calculation applied to all body regions. The current algorithm does not use any segmentations of particular organ or body composition. Instead, the segmentation is carried out overall patient image, and the SD map is calculated for the whole image; in addition, the magnitude of noise is simply determined in the most homogeneous area.

\section{Material and Methods}

\section{Algorithm of automated noise cal- culation}

In this retrospective study, noise is generally determined by calculating the standard deviation (SD) within a homogeneous area in the region of interest (ROI). Automated noise calculations require the ROI determination to be done automatically. The algorithm of automatic noise calculation in the proposed study consists of some steps as follows: 1) Segmenting images using an algorithm proposed previously by Anam et al. [25], and converting of the pixel values outside the image of patient (Figure 1a) to be 0 (zero) value.

$I_{b}(x, y)=\left\{\begin{array}{c}I_{o}(x, y), \text { inside the patient } \\ 0, \text { otherwise }\end{array}\right.$

The result of segmentation and conversion of the background values to 0 is shown in Figure $1 \mathrm{~b}$. This ensures that the area outside the patient is truly homogeneous.

2) Calculating the SD value for each pixel with a sliding window operation is performed using a specific kernel size. The SD value is calculated using the equation for each pixel:

$$
S D_{o}(x, y)=\sqrt{\frac{1}{n \times n} \sum_{i=1}^{n \times n}\left(I_{b, i}-\overline{I_{b}}\right)^{2}}
$$

Where $n \times n$ is the size of the sliding win-
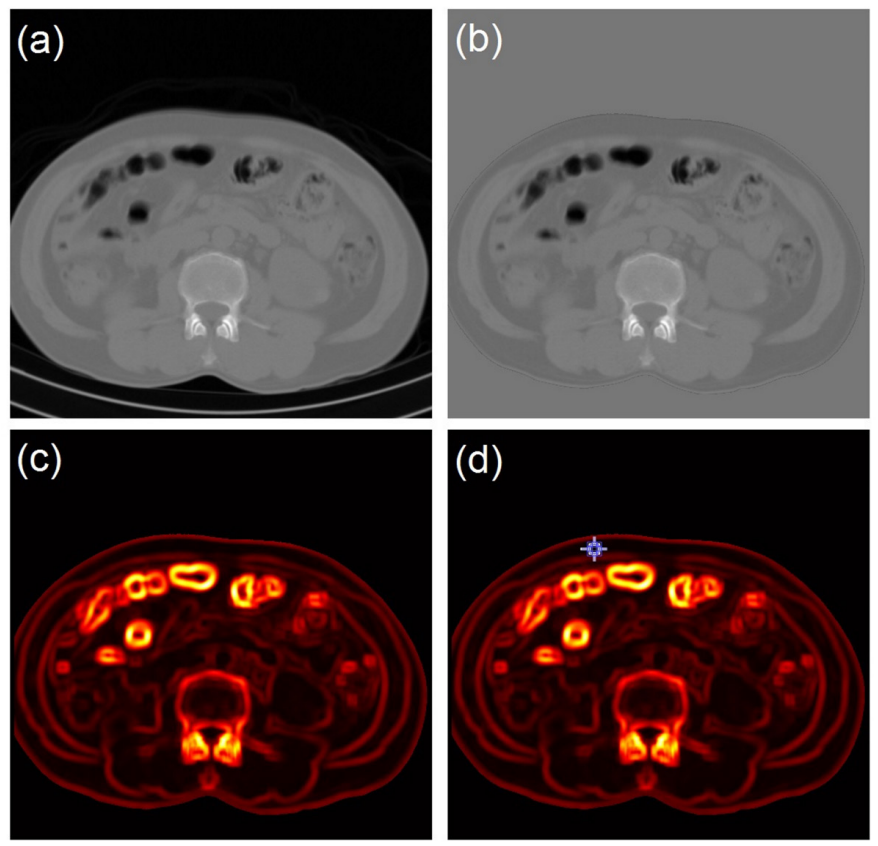

Figure 1: The steps of algorithm of automated noise calculation. (a) Original computerized tomography (CT) image, (b) Image of patient after converting area outside the patient to zero value, (c) Image of the standard deviation (SD) map, (d) The region of interest (ROI) for noise calculation is located in the most homogeneous area of image, i.e. the smallest value of the SD map. 
dow. After the SD calculation is completed in one pixel, the window shifts to next one pixel for calculating the SD value as before. The process is repeated to calculate the SD values for all pixels. Based on this process, the SD map is obtained. An example of the SD map is shown in Figure 1c. The effect of kernel size was also examined on the magnitude of noise for a homogeneous phantom.

3) The estimation of noise magnitude from the SD maps is determined in the most homogeneous areas. The determination of the most homogeneous area from the SD map is easy to do, i.e. the area with the smallest SD value. It should be noted that we expect the smallest value of SD to be within the patients, not outside them. Therefore, the value of the SD outside the patient is set to $100 \mathrm{HU}$ to ensure that the measured smallest SD is inside the patient.

$$
S D_{n}(x, y)=\left\{\begin{array}{l}
100, \text { if } S D_{0}(x, y) \leq 0.5 \\
S D_{0}(x, y), \text { otherwise }
\end{array}\right.
$$

A value of $0.5 \mathrm{HU}$ is chosen because it is almost predictable that the SD values are zero outside of patients (as described earlier); while there is no $\mathrm{SD}$ value smaller than 0.5 $\mathrm{HU}$ within patients or phantoms. After the SD background value is converted to 100 , the SD values are sorted from smallest to largest, and then the smallest SD value is taken. The location of the smallest value of SD indicates the most homogeneous area, and this smallest SD value is considered the noise magnitude of an image.

$$
\sigma=\min \left(S D_{n}(x, y)\right)
$$

The smallest SD position is determined by the equation:

$$
\left[x_{\min }, y_{\min }\right]=\operatorname{argmin}\left(S D_{n}\right)
$$

An example of ROI in the most homogeneous area is shown in the blue box in Figure 1d. For evaluation, we also tried to compare the noise magnitude from the one of the smallest SD with a mean of 2 smallest SDs up to a mean of 10 smallest SDs. The value of the average $\mathrm{N}$ smallest $\mathrm{SD}$ is calculated by the equation:

$$
\bar{\sigma}=\frac{1}{N} \sum_{i=1}^{N} \sigma_{i}
$$

Phantoms, patients, and data acquisition

To test the algorithm of the automated noise calculation, the algorithm was applied to images of a homogenous phantom (Toshiba Medical Systems Ltd., Tokyo, Japan) (Figure 2), 27 abdominal images of patients, and an adult anthropomorphic phantom (Kyoto Kagaku Ltd., Kyoto, Japan) (Figure 2).

Figure $2 \mathrm{a}$ and $\mathrm{b}$ show a photograph of homogeneous phantom and an axial image of
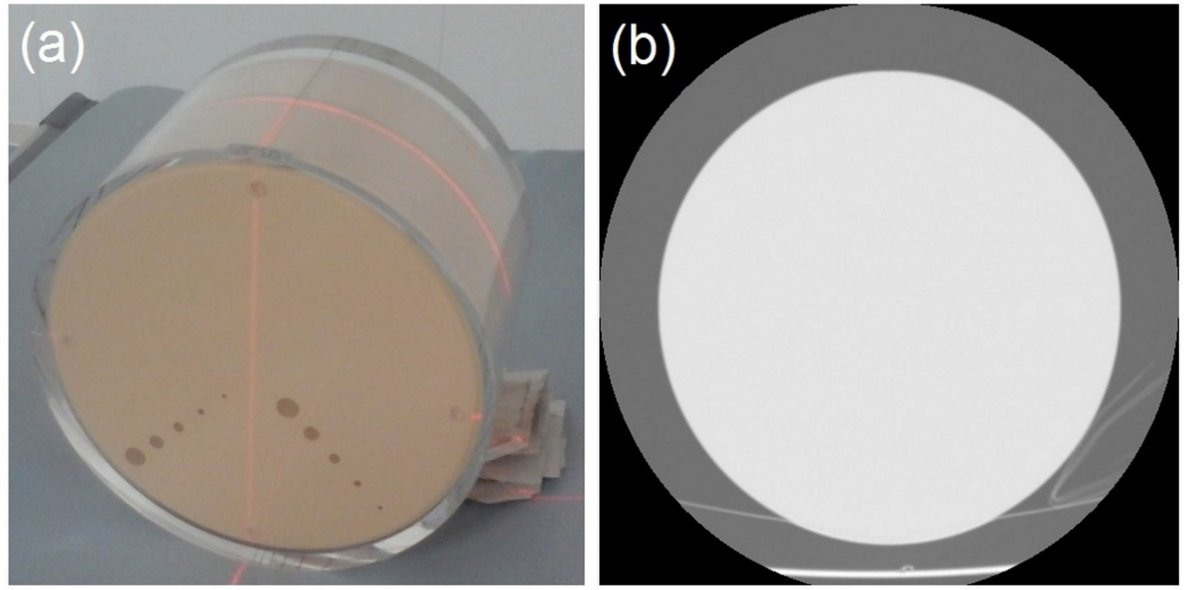

Figure 2: (a) Homogeneous phantom, (b) an axial image of the phantom. 
the phantom, respectively. The phantom was scanned using a MSCT Toshiba Alexion ${ }^{\mathrm{TM}} 4$ using parameters, including $120-\mathrm{kVp}$ tube voltage, $5 \mathrm{~mm}$ slice thickness, $1 \mathrm{~s}$ rotation time, 25 $\mathrm{cm}$ field of view (FOV), and 1.375 pitch. The algorithm was applied to the images of the homogenous phantom with different kernel sizes ranging from about $2 \mathrm{~mm} \times 2 \mathrm{~mm}$ to about $15 \mathrm{~mm} \times 15 \mathrm{~mm}$, and different numbers $(\mathrm{N})$ of ROIs ranging from 1 to 10 . The phantom was also scanned using different tube currents ranging from $25 \mathrm{~mA}$ to $150 \mathrm{~mA}$, and images were reconstructed with the three-dimensional adaptive iterative dose reduction (AIDR 3D) algorithm using different noise suppression levels (weak, mild, standard and strong).

Our algorithm was applied retrospectively to abdominal images of 27 patients scanned using a Siemens Emotion 6 CT Scanner, and reconstructed by SyngoCT 2006A software. The input parameters of the scans were 130 $\mathrm{kVp}, 0.6 \mathrm{~s}$ rotation times, tube current modulation with a reference of $95 \mathrm{mAs}, 2 \mathrm{~mm}$ slice thickness, and field of view (FOV), ranging from 253-370 $\mathrm{mm}$. The noise results of the proposed method were compared to those of a previous method [20].

The anthropomorphic phantom was scanned from underneath the pelvis to the apex of the head. Figure 3a-c shows the adult anthropomorphic phantom, a CT localizer radiograph of the phantom in the anterior-posterior (AP) direction and axial images of the phantom, respectively. The phantom was scanned using a MSCT Toshiba Alexion ${ }^{\mathrm{TM}}$ 4. The phantom was scanned to get a CT localizer radiograph, prior to helical scanning from the apex of the head to the bottom of the pelvis using a fixed tube currents $(25,50$, and $100 \mathrm{mAs})$ along the z-axis. The axial images were reconstructed using method of the filtered back projection (FBP) with a FC13 reconstruction filter. Scans were performed with a pitch of 1.5 , a rotation time of $1 \mathrm{~s}$, field of view (FOV) of $40 \mathrm{~cm}$, slice thickness of $7 \mathrm{~mm}$, and "large" filter type.

We calculated the noise automatically us-
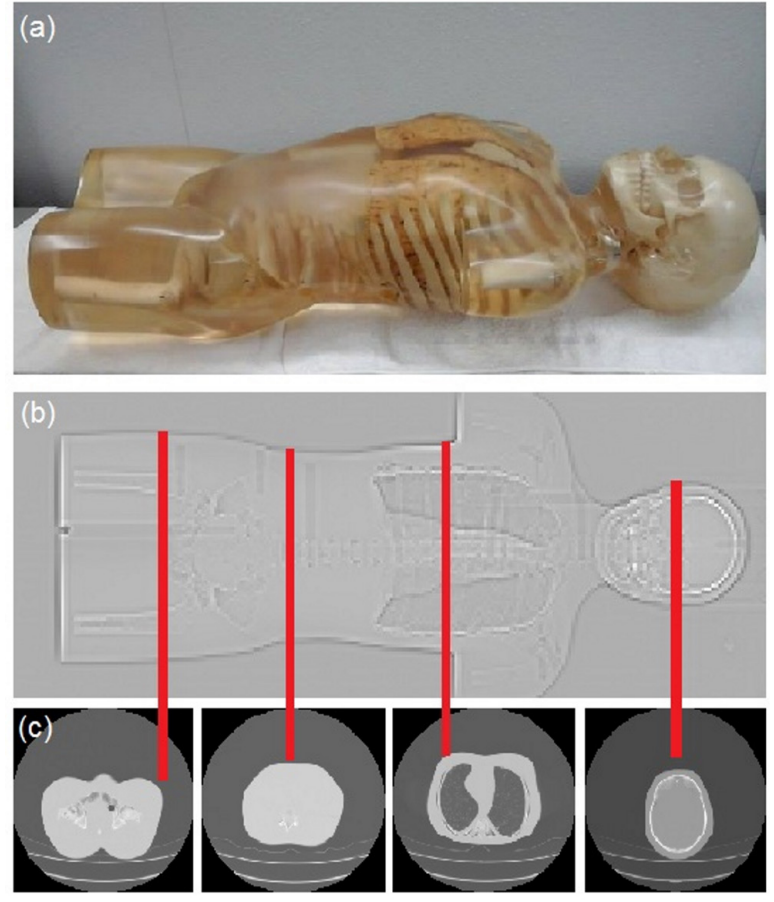

Figure 3: (a) An anthropomorphic adult male phantom, (b) localizer radiograph in the anterior-posterior (AP) direction, (c) axial images for head, thorax, abdomen and pelvis images.

ing our proposed algorithm and calculated $\mathrm{D}_{\mathrm{w}}$ in the axial image of each slice automatically using an algorithm proposed previously [25] based on following equation:

$$
D_{w}=2 \sqrt{\left[\frac{1}{1000} \overline{H U}+1\right] \frac{A_{R O I}}{\pi}}
$$

Where $\mathrm{A}_{\mathrm{ROI}}$ and $\overline{H U}$ are the area of the phantom after contouring and the average $\mathrm{HU}$ value of the phantom, respectively. The image noise of each slice should correlate with $\mathrm{D}_{\mathrm{w}}$ value. Based on the comparison of the noise level and the calculated $\mathrm{D}_{\mathrm{w}}$ for every slice, the accuracy of the automated noise algorithm can be established.

\section{Results}

Noise magnitude in the homogeneous phantom images

Determination of automatic noise based on 
the smallest SD value is probably a random event. To test this, the average noise up to $\mathrm{N}$ $=10$ of the smallest SD values was evaluated and the results are shown in Figure 4 (a). The noise value rises linearly $\left(\mathrm{R}^{2}=0.9897\right)$ with the number, N. The difference based on using one of the smallest SD and the average of the $\mathrm{N}=10$ smallest SDs is still below $1.0 \%$ as shown in Figure 4 (b). Thus, the use of only the smallest SD is a good descriptor for the noise of an image. The noise value is probably
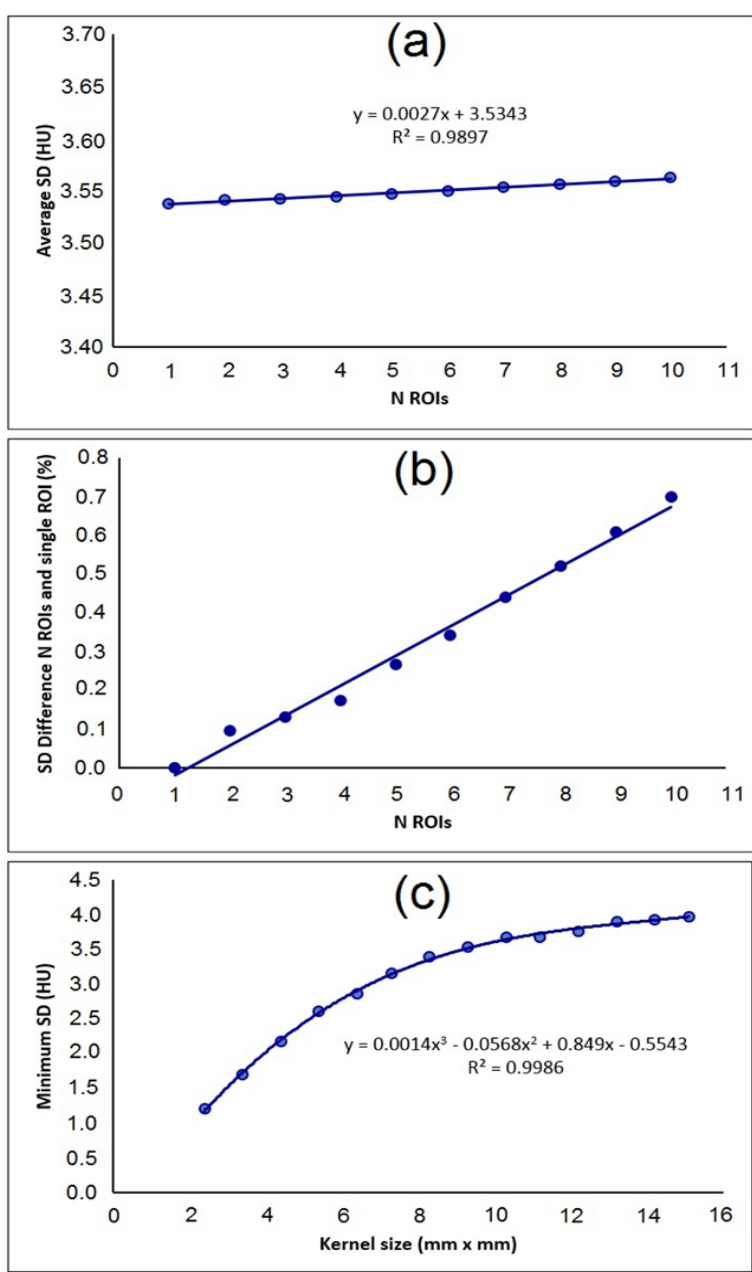

Figure 4: (a) The noise of average $\mathrm{N}$ smallest standard deviations (SDs), (b) The percentage difference of noise in the use of one of region of interest (ROI) and N ROIs, and (c) The noise magnitude of a homogeneous phantom as a function of the ROI size (or kernel size). determined by the size of the ROI or the sliding window. Figure $4 \mathrm{c}$ confirms that the noise value of a homogeneous phantom increases as the size ROI increases from around $2 \mathrm{~mm} \times 2$ $\mathrm{mm}$ to $15 \mathrm{~mm} \times 15 \mathrm{~mm}$.

The relationships between the minimum SD, the tube current and type of noise suppression in AIDR 3D image reconstruction are shown in Figure 5. Figure 5a shows that the noise decreases with the increase in tube current; in addition, Figure $5 \mathrm{~b}$ shows that the noise magnitude depends on the type of noise suppression. As expected, AIDR 3D with stronger noise suppression settings results in less noise than AIDR 3D standard, and AIDR with the weaker suppression results in more noise than the standard one.

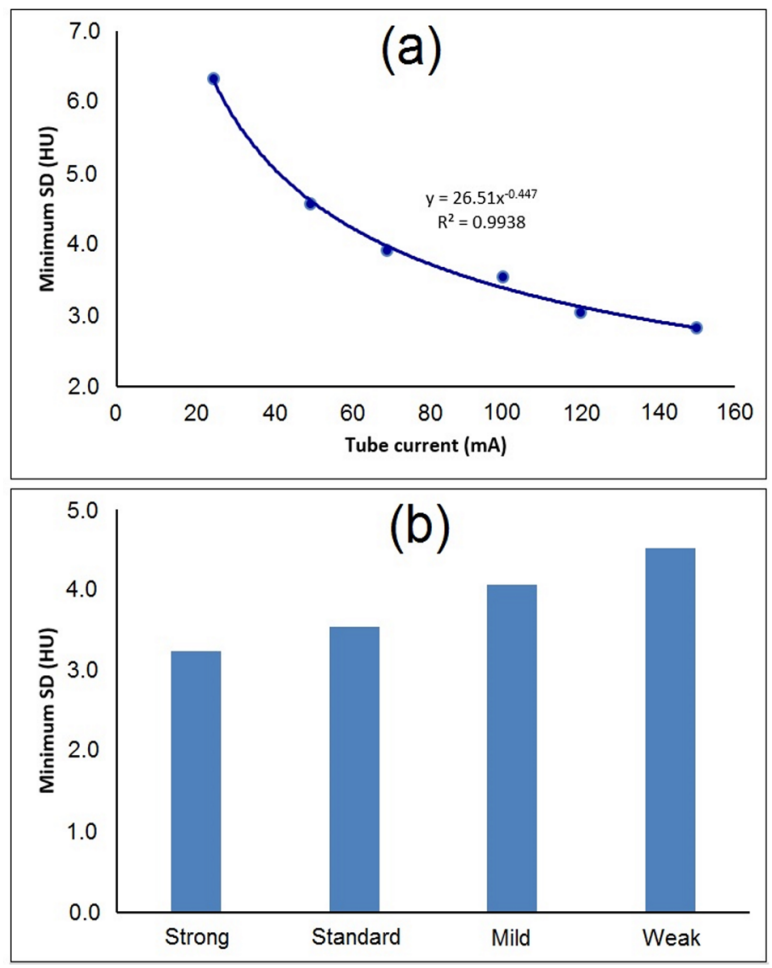

Figure 5: (a) The relationships between noise magnitude and tube current in the homogeneous phantom, and (b) The graph showing noise magnitude for different types of noise suppression of the adaptive iterative dose reduction (AIDR 3D) image reconstruction, i.e. strong, standard, mild and weak ones. 
Comparison of the proposed algorithm and previous algorithm

To validate the results of the proposed algorithm, it was compared with those from the previous algorithm [20]. The comparison was carried out on the homogeneous phantom and 27 abdominal images (for the patient images except the abdomen, comparison cannot be made because the previous algorithm was only applicable to the abdomen where soft tissue is the majority component). The comparison of the noise magnitude from the proposed and previous methods in the homogeneous phantom is shown in Figure 6a. There is a linear correlation between both with an $\mathrm{R}^{2}$ value of 0.9968 . The noise magnitude from the proposed method is about half of the value of the global noise measured from the previous method. The box-whisker graphs for
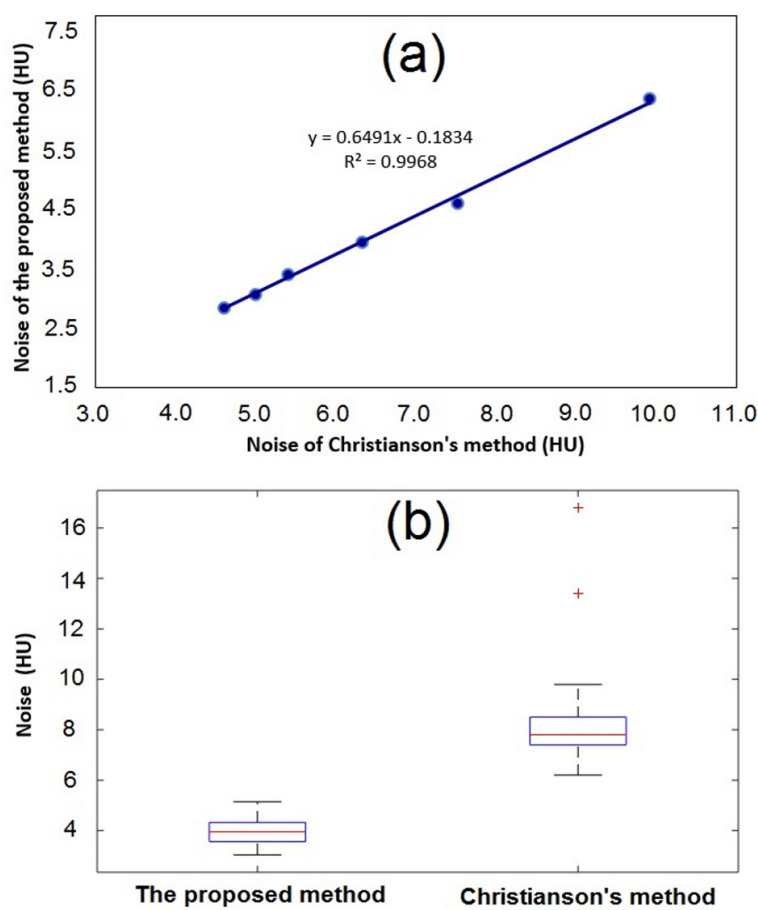

Figure 6: (a) The relationship between the noise magnitude from the proposed method and the previous method in the homogeneous phantom, and (b) The box-whisker graph for noise magnitude from the proposed method and previous method in the abdominal image. noise magnitude measured using the proposed and the previous methods in the abdominal image are shown in Figure 6b, which clearly show that the noise magnitude of our proposed method is about half the global noise measured from the previous method. The global noise from the previous method has two outlier data, i.e. 13.4 HU and 16.8 HU, while our proposed method has no outlier data.

\section{Automated noise calculation on the} whole body anthropomorphic phantom

The noise magnitudes calculated by the proposed method for three fixed tube currents, including 25, 50 and $100 \mathrm{~mA}$ on full body of anthropomorphic phantom are shown in Figure $7 \mathrm{a}$, and the box-whisker graphs of noise are shown in Figure $7 \mathrm{~b}$. These show that the proposed algorithm can distinguish the mag-
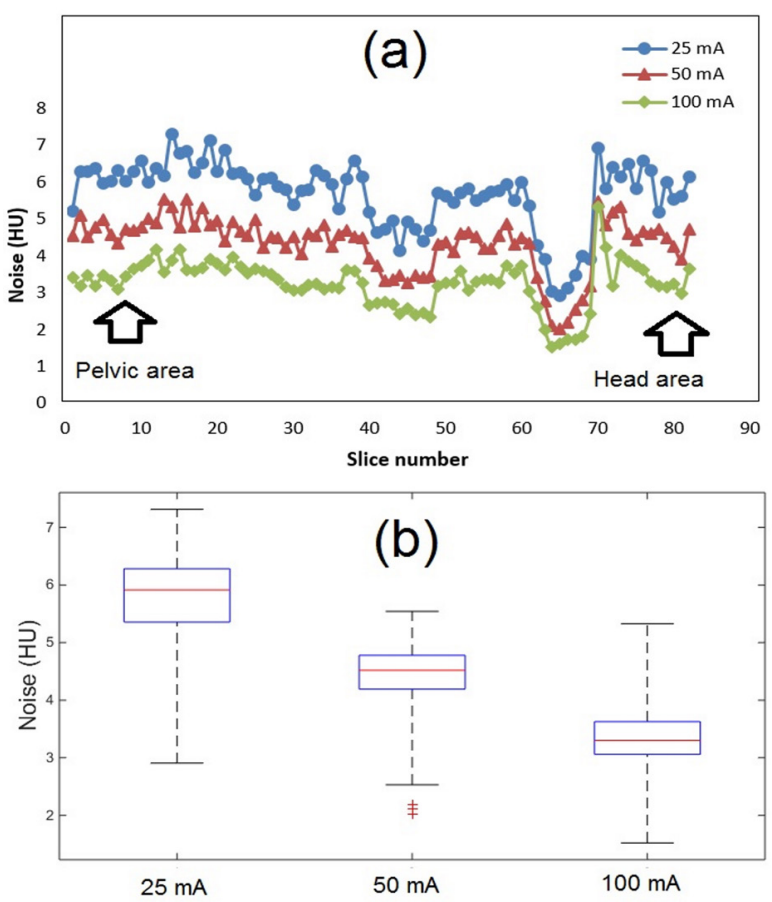

Figure 7: (a) The noise magnitude calculated by the proposed method overall anthropomorphic phantom for three fixed tube currents $(25,50$ and $100 \mathrm{~mA})$, and (b) The boxwhisker graphs of noise from the completely anthropomorphic phantom for three fixed tube currents. 
nitude of noise for variations due to different tube currents from head to pelvis of the anthropomorphic phantom.

The dependencies of the noise values and the water-equivalent diameter $\left(D_{w}\right)$ from the head to the bottom of the pelvis are shown in Figure 8 for three fixed tube currents. It is seen that the noise magnitude closely follows the magnitude of $\mathrm{D}_{\mathrm{w}}$. In the pelvic and abdominal area, noise is relatively high because the $\mathrm{D}_{\mathrm{w}}$ value is also relatively high; in addition, in the area of the thorax, noise decreases because $\mathrm{D}_{\mathrm{w}}$ also decreases (lung area), and in the upper thoracic region, noise rises again since $\mathrm{D}_{\mathrm{w}}$ rises (the presence of the phantom arm). Moreover, in the neck area, the noise decreases because the $\mathrm{D}_{\mathrm{w}}$ is small, and finally the noise in the head area rises again because $\mathrm{D}_{\mathrm{w}}$ rises. The increase of noise magnitude in the head area, especially at the bottom of the head area, exceeds the increase of $\mathrm{D}_{\mathrm{w}}$. All patterns were observed at a fixed current of $25 \mathrm{~mA}$ (Figure 8a, $50 \mathrm{~mA}$ (Figure 8b) and $100 \mathrm{~mA}$ (Figure 8c).

\section{Discussion}

Automatic noise calculation performed directly from the patient image is an important factor to optimize the radiation dose received by the patient. Automated dose calculation received by patients using the concept of size-specific dose estimate (SSDE) has been widely reported [15, 26-28]. While automated noise calculations performed directly from patient images have been reported previously $[20,24]$, they have been limited to certain body parts such as the abdomen or thorax. Our proposed algorithm is an improvement of the algorithm proposed previously [20, 24], such that it can be applied to calculate noise in all body regions without overestimating the predicted noise.

Automatic noise calculation in our proposed method is based on the fact that noise is usually measured in the most homogeneous area. The most homogeneous area can be easily determined as the position of the smallest SD on
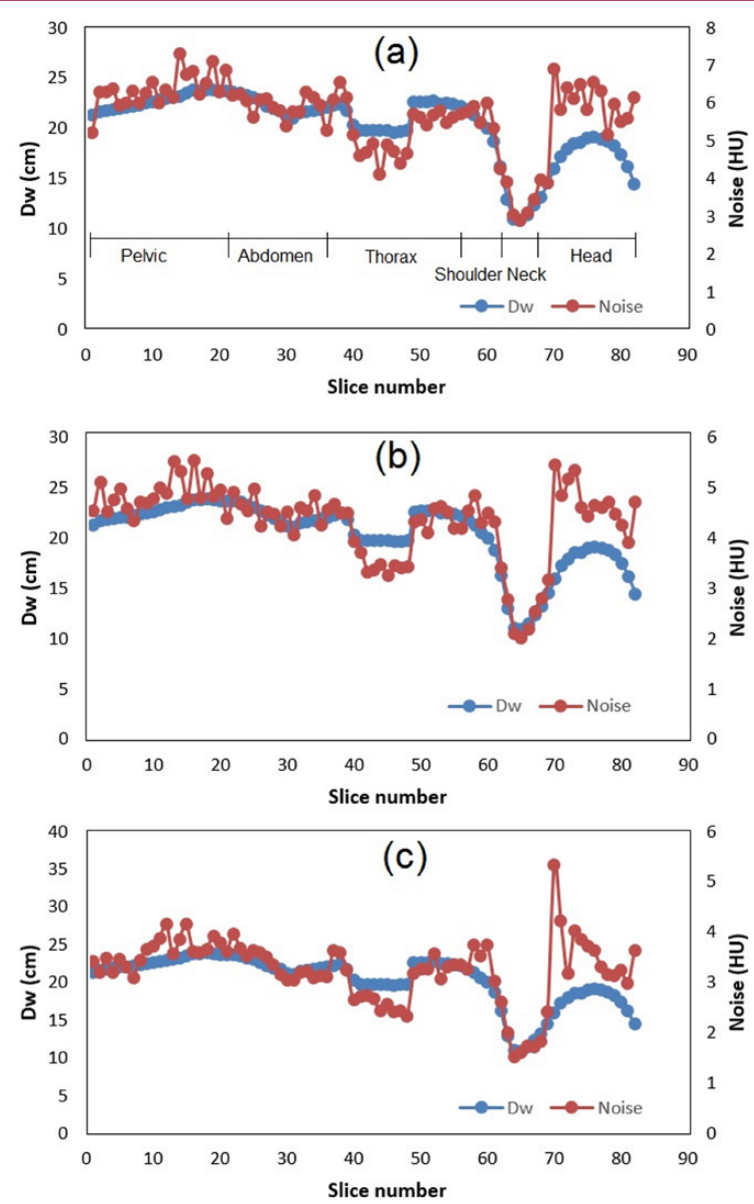

Figure 8: The noise and the water-equivalent diameter $\left(D_{w}\right)$ along the $z$-axis from the head to the bottom of the pelvis. (a) $25 \mathrm{~mA}$, (b) 50 $\mathrm{mA}$, and (c) $100 \mathrm{~mA}$.

the SD map of an image and noise can be identified as the smallest SD itself. The noise can be estimated by not only one of the smallest $\mathrm{SD}$ values but also, for example, the average of 5 or 10 of the smallest SDs. The data obtained in this study shows that based on using the average of up to 10 of the smallest SDs, the noise is within $1 \%$ of the absolute smallest $\mathrm{SD}$. The calculation of SD in a pixel is certainly influenced by the size of the kernel of sliding window (ROI size) so that the SD value increases if the size of the sliding window increases, as confirmed in this study. The size of the ROI must be consistent. We have followed the AAPM recommendation of using an ROI of $10 \mathrm{~mm} \times 10 \mathrm{~mm}$ [29] for noise calculation. 
Automated noise calculation must be able to distinguish noise magnitude for different input parameters. Our proposed algorithm is able to distinguish the noise magnitude due to variations in tube currents and different levels of noise suppression in a reconstructed image using the AIDR 3D algorithm, as reported previously by Ang et al. [30] using the manual method. There is a linear correlation between the noise calculated automatically using our proposed algorithm and the previous algorithm [20] for homogeneous phantoms. The noise magnitude measured using the proposed algorithm is about half of that calculated using the earlier algorithm [20]. Since the previous algorithm measured global noise, the noise magnitude is approximately the same as the noise magnitude measured by the subtraction method, which is known to be about twice the actual noise in an image [21-23].

The results of the earlier automated noise calculation [20] are quite consistent with others if the soft-tissue composition of the abdomen was relatively dominant. However, in any other cases, for example when soft tissue is not dominant or localized in a sufficient area, that method was not able to predict noise accurately. From 27 abdominal patient images, there were two images where that method did not successfully predict noise. Based on the use of our novel proposed method, this did not happen. Our novel algorithm remains accurate even when the composition of the soft tissue in the image is small or non-localized.

We found that our proposed method could be applied to all body regions, from the head to the bottom of the pelvis. For example, for the anthropomorphic phantom Figure 8 shows that, the noise magnitude corresponds to the magnitude of the water equivalent diameter $\left(D_{w}\right)$ and this result is consistent with previous studies $[12,31]$. A slightly different situation is seen in the head region because there are many bones in the head so that the result of beam hardening artifact causes noise in the head to be slightly larger than that outside the head re- gion [32]. An anthropomorphic head image is shown in Figure 9a, with its SD map shown in Figure 9b. For comparison, Figure 9 c shows the SD map obtained from the previous algorithm [20]. There is almost no soft tissue with HU value from 0-100 and the segmented areas are only in the boundary between the head and the air outside of the head. Thus, the corresponding SD histogram shown in Figure 9d does not display any the actual global noise values. It shows a global noise value of around $41 \mathrm{HU}$, while based on our proposed method, the value is around $5 \mathrm{HU}$, i.e. just higher than the expected value. Thus, our proposed method can be implemented in the head region.

The results show that our proposed method can accurately calculate noise automatically in clinical images of patients. If the automated noise calculation method is combined with automated spatial resolution determination [14, 33] and automated organ HU measurement [34], the quantization of patient image quality can be accomplished more comprehensively. Furthermore, by combining automated quantification of image quality with automated patient dose calculation in a SSDE metric [35, 36], there would be opportunities to optimize the CT protocol more intuitively.

This study has several limitations as follows: first, implementation of the proposed method in the head, thorax and pelvic images of patients has not been carried out. The implementation is only carried out on an anthropomorphic phantom. Implementation of our automated noise calculation on different body parts, especially in the head region, and comparison with results obtained from the observation of radiologist experts still has needed to be carried out. Second, in this study, the results of automated noise calculation have not been compared with the results of patient dose for dose optimization process. In a forthcoming study, a direct comparison between patient dose and image quality will be investigated, for example using the figure of merit (FOM) approach [37]. 

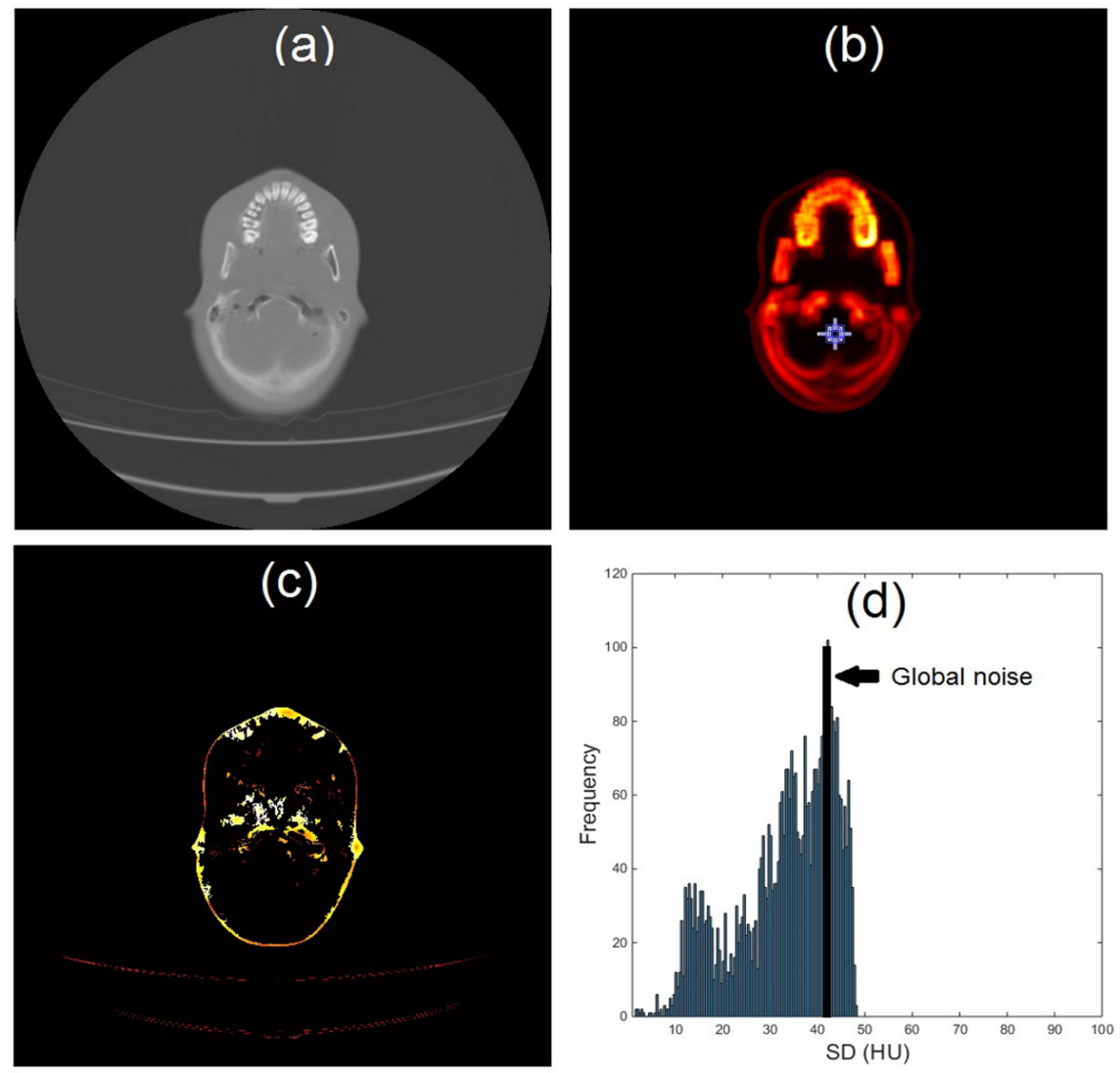

Figure 9: (a) Example of anthropomorphic head image for automated noise calculation, (b) The standard deviation (SD) map along with region of interest (ROI) position using our proposed algorithm, (c) The SD map using previous algorithm, (d) Corresponding SD histogram and value of global noise is about 41 Hounsfield unit (HU).

\section{Conclusion}

We successfully developed an automated noise methodology implemented in CT images of any regions of the body. It was successfully implemented in images of a homogeneous phantom, an anthropomorphic phantom, and several patient images. Our method is able to distinguish the noise magnitude due to variations in input parameters such as tube currents or image reconstruction algorithms. As expected, in the abdominal patient images, the noise measured using our method is about half of the global noise. Our method produces noise magnitudes, which closely follow the magnitudes of the water equivalent diameter in all body regions. The method is easily implemented, robust, and accurate. It could potentially be used to optimize CT protocols. Implementation of automated noise calculation along with automated size-specific estimation may provide a convenient tool for optimizing patient doses according to the ALARA principle.

\section{Acknowledgment}

This work was funded by the Penelitian Dasar Unggulan Perguruan Tinggi (PDUPT) 2019, Ministry of Research Technology and Higher Education of the Republic of Indonesia. The authors give special thanks to $\mathrm{Mr}$. Masdi from Prof. Dr. Margono Hospital for providing images of the patients.

\section{Conflict of Interest}

None 
Automated Noise Measurement in CT

\section{References}

1. Brenner D, Elliston C, Hall E, Berdon W. Estimated risks of radiation-induced fatal cancer from pediatric CT. AJR Am J Roentgenol. 2001;176:289-96. doi: 10.2214/ajr.176.2.1760289. PubMed PMID: 11159059 .

2. Mathews JD, Forsythe AV, Brady Z, Butler MW, Goergen SK, Byrnes GB, et al. Cancer risk in 680,000 people exposed to computed tomography scans in childhood or adolescence: data linkage study of 11 million Australians. BMJ. 2013;346:f2360. doi: 10.1136/bmj.f2360. PubMed PMID: 23694687. PubMed PMCID: PMCPMC3660619.

3. Miglioretti DL, Johnson E, Williams A, Greenlee RT, Weinmann S, Solberg LI, et al. The use of computed tomography in pediatrics and the associated radiation exposure and estimated cancer risk. JAMA Pediatr. 2013;167:700-7. doi: 10.1001/jamapediatrics.2013.311. PubMed PMID: 23754213. PubMed PMCID: PMCPMC3936795.

4. Pearce MS, Salotti JA, Little MP, McHugh K, Lee C, $\mathrm{Kim} \mathrm{KP}$, et al. Radiation exposure from CT scans in childhood and subsequent risk of leukaemia and brain tumours: a retrospective cohort study. Lancet. 2012;380:499-505. doi: 10.1016/S01406736(12)60815-0. PubMed PMID: 22681860. PubMed PMCID: PMCPMC3418594.

5. Kalender WA. Dose in x-ray computed tomography. Phys Med Biol. 2014;59:R129-50. doi: 10.1088/0031-9155/59/3/R129. PubMed PMID: 24434792.

6. Kalra MK, Maher MM, Toth TL, Hamberg LM, Blake MA, Shepard JA, et al. Strategies for CT radiation dose optimization. Radiology. 2004;230:619-28. doi: 10.1148/radiol.2303021726. PubMed PMID: 14739312.

7. Larson DB. Optimizing CT radiation dose based on patient size and image quality: the size-specific dose estimate method. Pediatr Radiol. 2014;44 Suppl 3:501-5. doi: 10.1007/s00247-014-3077-y. PubMed PMID: 25304711.

8. Verdun F, Meuli R, Bucher G, Noel A, Stines J, Schnyder $P$, et al. Dose and image quality characterisation of CT units. Radiation Protection Dosimetry. 2000;90:193-6. doi: 10.1093/0xfordjournals.rpd.a033117.

9. Goldman LW. Principles of CT: radiation dose and image quality. J Nucl Med Technol. 2007;35:21325. doi: 10.2967/jnmt.106.037846. PubMed PMID: 18006597.

10. Garayoa J, Castro P. A study on image quality provided by a kilovoltage cone-beam computed to- mography. J Appl Clin Med Phys. 2013;14:3888. doi: 10.1120/jacmp.v14i1.3888. PubMed PMID: 23318380. PubMed PMCID: PMCPMC5714052.

11. Roa AM, Andersen HK, Martinsen AC. CT image quality over time: comparison of image quality for six different CT scanners over a six-year period. $J$ Appl Clin Med Phys. 2015;16:4972. doi: 10.1120/ jacmp.v16i2.4972. PubMed PMID: 26103172. PubMed PMCID: PMCPMC5690105.

12. Larson DB, Malarik RJ, Hall SM, Podberesky DJ. System for verifiable CT radiation dose optimization based on image quality. part II. process control system. Radiology. 2013;269:177-85. doi: 10.1148/radiol.13122321. PubMed PMID: 23784877.

13. Larson DB, Wang LL, Podberesky DJ, Goske MJ. System for verifiable CT radiation dose optimization based on image quality. part I. Optimization model. Radiology. 2013;269:167-76. doi: 10.1148/radiol.13122320. PubMed PMID: 23784878.

14. Sanders J, Hurwitz L, Samei E. Patient-specific quantification of image quality: An automated method for measuring spatial resolution in clinical CT images. Med Phys. 2016;43:5330. doi: 10.1118/1.4961984. PubMed PMID: 27782718.

15. Dodge CT, Tamm EP, Cody DD, Liu X, Jensen CT, Wei $W$, et al. Performance evaluation of iterative reconstruction algorithms for achieving CT radiation dose reduction - a phantom study. J Appl Clin Med Phys. 2016;17:511-31. doi: 10.1120/ jacmp.v17i2.5709. PubMed PMID: 27074454. PubMed PMCID: PMCPMC5875046.

16. Hussain FA, Mail N, Shamy AM, Suliman A, Saoudi A. A qualitative and quantitative analysis of radiation dose and image quality of computed tomography images using adaptive statistical iterative reconstruction. J Appl Clin Med Phys. 2016;17:419-32. doi: 10.1120/jacmp.v17i3.5903. PubMed PMID: 27167261. PubMed PMCID: PMCPMC5690909.

17. Kijewski MF, Judy PF. The noise power spectrum of CT images. Phys Med Biol. 1987;32:565-75. doi: 10.1088/0031-9155/32/5/003. PubMed PMID: 3588670.

18. Pineda AR, Tward DJ, Gonzalez A, Siewerdsen $\mathrm{JH}$. Beyond noise power in 3D computed tomography: the local NPS and off-diagonal elements of the Fourier domain covariance matrix. Med Phys. 2012;39:3240-52. doi: 10.1118/1.4705354. PubMed PMID: 22755707. PubMed PMCID: PMCPMC3365918. 
19. Nakahara S, Tachibana M, Watanabe Y. One-year analysis of Elekta CBCT image quality using NPS and MTF. J Appl Clin Med Phys. 2016;17:211-22. doi: 10.1120/jacmp.v17i3.6047. PubMed PMID: 27167279. PubMed PMCID: PMCPMC5690923.

20. Christianson O, Winslow J, Frush DP, Samei E. Automated Technique to Measure Noise in Clinical CT Examinations. AJR Am J Roentgenol. 2015;205:W93-9. doi: 10.2214/AJR.14.13613. PubMed PMID: 26102424.

21. Friedman SN, Fung GS, Siewerdsen JH, Tsui BM. A simple approach to measure computed tomography (CT) modulation transfer function (MTF) and noise-power spectrum (NPS) using the American College of Radiology (ACR) accreditation phantom. Med Phys. 2013;40:051907. doi: 10.1118/1.4800795. PubMed PMID: 23635277. PubMed PMCID: PMCPMC3643984.

22. Dolly S, Chen HC, Anastasio M, Mutic S, Li H. Practical considerations for noise power spectra estimation for clinical CT scanners. J Appl Clin Med Phys. 2016;17:392-407. doi: 10.1120/jacmp. v17i3.5841. PubMed PMID: 27167257. PubMed PMCID: PMCPMC5690921.

23. Siewerdsen JH, Cunningham IA, Jaffray DA. A framework for noise-power spectrum analysis of multidimensional images. Med Phys. 2002;29:2655-71. doi: 10.1118/1.1513158. PubMed PMID: 12462733.

24. Chun M, Choi YH, Kim JH. Automated measurement of CT noise in patient images with a novel structure coherence feature. Phys Med Biol. 2015;60:9107-22. doi: 10.1088/00319155/60/23/9107. PubMed PMID: 26561914.

25. Anam C, Haryanto F, Widita R, Arif I, Dougherty G. Automated Calculation of Water-equivalent Diameter (DW) Based on AAPM Task Group 220. J Appl Clin Med Phys. 2016;17:320-33. doi: 10.1120/jacmp.v17i4.6171. PubMed PMID: 27455491. PubMed PMCID: PMCPMC5690059.

26. Sarmento S, Mendes B, Gouvea M. Automatic calculation of patient size metrics in computed tomography: What level of computational accuracy do we need? J Appl Clin Med Phys. 2018;19:21827. doi: 10.1002/acm2.12240. PubMed PMID: 29265700. PubMed PMCID: PMCPMC5768030.

27. Christianson O, Li X, Frush D, Samei E. Automated size-specific CT dose monitoring program: assessing variability in CT dose. Med Phys. 2012;39:7131-9. doi: 10.1118/1.4761871. PubMed PMID: 23127104.
28. Anam C, Haryanto F, Widita R, Arif I, Dougherty G. A fully automated calculation of size-specific dose estimates (SSDE) in thoracic and head CT examinations. J Phys Conf Ser. 2016;694:012030. doi: 10.1088/1742-6596/694/1/012030.

29. Committee ADX-R. Specification and acceptance testing of computed tomography scanners. Report no. 39; New York: American Association of Physicists in Medicine; 1993.

30. Ang WC, Hashim S, Karim MKA, Bahruddin NA, Salehhon N, Musa Y, et al. Adaptive iterative dose reduction (AIDR) 3D in low dose CT abdomenpelvis: Effects on image quality and radiation exposure. J Phys Conf Ser. 2017;851:012006. doi: 10.1088/1742-6596/851/1/012006.

31. Tian X, Samei E. Accurate assessment and prediction of noise in clinical CT images. Med Phys. 2016;43:475. doi: 10.1118/1.4938588. PubMed PMID: 26745940.

32. Barrett JF, Keat N. Artifacts in CT: recognition and avoidance. Radiographics. 2004;24:167991. doi: 10.1148/rg.246045065. PubMed PMID: 15537976.

33. Anam C, Fujibuchi T, Budi WS, Haryanto F, Dougherty G. An algorithm for automated modulation transfer function measurement using an edge of a PMMA phantom: Impact of field of view on spatial resolution of CT images. J Appl Clin Med Phys. 2018;19:244-52. doi: 10.1002/acm2.12476. PubMed PMID: 30338920. PubMed PMCID: PMCPMC6236841.

34. Abadi E, Sanders J, Samei E. Patient-specific quantification of image quality: An automated technique for measuring the distribution of organ Hounsfield units in clinical chest CT images. Med Phys. 2017;44:4736-46. doi: 10.1002/mp.12438. PubMed PMID: 28658516.

35. Anam C, Haryanto F, Widita R, Arif I, Dougherty G. The Size-Specific Dose Estimate (Ssde) for Truncated Computed Tomography Images. Radiat Prot Dosimetry. 2017;175:313-20. doi: 10.1093/ rpd/ncw326. PubMed PMID: 27885082.

36. Anam C, Arif I, Haryanto F, et al. A simplified method for the water-equivalent diameter calculation to estimate patient dose in CT examinations. Radiat Prot Dosim. 2019;185:42-9. doi: 10.1093/ rpd/ncy214. PubMed PMID: 30508150.

37. Pace E, Borg M. Optimisation of a Paediatric Ct Brain Protocol: A Figure-of-Merit Approach. Radiat Prot Dosimetry. 2018;182:394-404. doi: 10.1093/rpd/ncy078. PubMed PMID: 29788223. 